\title{
LA MONACAZIONE DEL LUPO NELLA COMPOSIZIONE DELLA BRANCHE 3 DEL RENART
}

\begin{abstract}
Tl testo della br. 3 del Roman de Renart è tràdito da tutti i testimoni noti, a parte K (Chantilly, Musée Condé, 472), ${ }^{1}$ ma a fronte di questa vasta diffusione mostra poche variazioni sostanziali tra i codici (illustrate in Harano 1999 e in parte discusse già in Rychner 1969) e una tradizione testimoniale quasi priva di contaminazioni. Ernest Martin ${ }^{2}$ fonda la sua edizione sul ms. A, attingendo a D per le sezioni mancanti, ed effettivamente va rilevato che per questa branche la famiglia $\alpha$ conserva tutta una serie di lectiones difficiliores che $\beta$ e $\gamma$ tendono a semplificare o espungere (se ne trovano esempi in Harano 1999: 41-3) e che invece incoraggiano «cette conviction que l'auteur de la branche III, très doué d'ailleurs, écrivait toujours mieux, d'une écriture plus pleine et plus relevée, que ses copistes successifs» (Rychner 1969: 133).
\end{abstract}

\footnotetext{
${ }^{1}$ Cioè tredici. Sono tutti i manoscritti della famiglia $\alpha$, vale a dire A (Paris, BnF, fr. 20043), in maniera frammentaria, D (Oxford, Bodleian Library, Douce 360), E (London, British Museum, Additional 15229), F (New York, Pierpont Morgan Library, M. 932) e G (Paris, BnF, fr. 1580), con la parziale eccezione di N (Vaticano, BAV, Reginensi Latini, 1699), che per la $b r .3$ segue il testo della famiglia $\gamma$ (divenendo $\mathrm{n}$ ); due testimoni su tre della famiglia $\beta$, ovvero $B$ (Paris, BnF, fr. 371) ed L (Paris, Bibliothèque de l'Arsenal, 3335); entrambi i testimoni della famiglia $\gamma$, ovvero C (Paris, BNF, fr. 1579) ed M (Torino, Biblioteca Reale, varia 151); nonché tutti e tre i testimoni cosiddetti compositi, H (Paris, Bibliothèque de l'Arsenal, 3334), I (Paris, BnF, fr., 12584) e O (Paris, BNF, fr. 12583). A questi vanno poi aggiunti il frammento s (Biblioteca pubblica di Siena, ms. K.IV.50), che contiene parti delle brs. 2, 3 e 4, e il frammento t (Hiroshima, University Library, 184624), che conserva parte della $b r .3$ e una porzione della $b r .2$.

2 Martin 1882-1887, su cui si basa anche Bonafin 2004, dal quale cito testo e traduzione della branche. Le altre traduzioni, ove non diversamente segnalato, sono mie; le citazioni di altre branches s'intendono sempre desunte dall'edizione Martin.
} 
Il tema della branche non è del tutto inedito. Due dei tre episodi che la compongono riprendono canovacci ampiamente diffusi a livello orale e non di rado narrati assieme. Nel primo, la volpe sottrae del cibo da un carretto di passaggio, fingendosi morta per lasciarsi caricare su dall'ingenuo guidatore, che vorrebbe rivenderne la pelle e invece viene derubato delle sue provviste. Il secondo descrive un inganno ordito dalla volpe ai danni di un altro animale - spesso, come in questo caso, il lupo -, che le chiede conto del cibo che si è procurata. La volpe gli indica un foro praticato su un lago ghiacciato, dal quale può pescare usando la coda. Col freddo, però, l'acqua in cui era immersa la coda si congela, condannando cosí l'animale a restare bloccato alla mercé di vari assalitori, dai quali sfugge solo al prezzo della perdita dell'arto. Tra questi due episodi, l'autore ne inserisce un altro che è invece estraneo alla tradizione popolare, vale a dire quello della monacazione del lupo. Mentre Renart sta cucinando il pesce che ha appena rubato, Isengrin, ammaliato dal profumo, bussa alla sua porta e chiede di poter unirsi alla sua mensa, ma Renart glielo impedisce, asserendo che quel cibo è riservato ai monaci che dimorano presso di lui. Il lupo, allora, insiste per ricevere anch'egli una tonsura e Renart ne approfitta per ingannarlo, facendogli infilare la testa in una fenditura e calandogli dell'acqua bollente addosso. La prova successiva per essere ammesso al convento sarà appunto quella della pesca nel lago ghiacciato, che causerà a Isengrin la perdita della sua coda.

Se i racconti del "furto del pesce" e della "pesca con la coda" appartengono al comune patrimonio folklorico, quello della monacazione del lupo è invece un tema di diversa provenienza, sviluppato in ambiente conventuale. ${ }^{3}$ Pertanto, la presenza di questo episodio diventa nodale, poiché è proprio la sua inserzione, all'interno di un intreccio potenzialmente autosufficiente, a dare la misura dell'intervento che l'autore ha compiuto sulla tradizione popolare cui ha attinto. Infatti, occorre precisare che dei due racconti sul furto e sulla pesca esistono molteplici versioni, in cui

${ }^{3}$ Una klösterliche tradition, che avrebbe sviluppato umoristicamente uno spunto tratto da Matteo 7,15: «Adtendite a falsis prophetis, qui veniunt ad vos in vestimentis ovium, intrinsecus autem sunt lupi rapaces» [Guardatevi dai falsi profeti, che vengono da voi vestiti da pecore ma dentro di loro sono lupi rapaci] (Voigt 1879: 290). 
cambiano il tipo di cibo rubato (ad es. formaggio o pollame), il modo in cui i due animali si incontrano e il secondo viene ingannato (ad es. la volpe può suggerire al lupo di imitarla ma quest'ultimo, fingendosi morto, viene bastonato), l'animale che pesca (originariamente non il lupo ma l'orso) e il metodo con cui lo fa (direttamente con la coda nell'acqua oppure legando alla coda un secchio). Soprattutto, i due racconti non sono necessariamente correlati tra loro, ma circolano anche autonomamente, o in sequenza con altri, che pure, in qualche caso, trovano riscontro in alcune branches renardiane (ad es. nell'intreccio della br. 4, Renart dans le puit). ${ }^{4} \mathrm{Da}$ questa grande varietà, l'autore della $b r .3$ ha selezionato due versioni precise, combinandole - è questa la tesi qui discussa - in funzione dell'inserzione del moniage Isengrin, che costituirebbe il vero fulcro attorno a cui ruota la costruzione dell'intera branche.

Poiché è l'episodio della monacazione del lupo che distingue la $b r .3$ dai tradizionali intrecci di matrice folklorica, questa aggiunta risulta altamente informativa del tipo di cultura che l'autore esprime, ovvero una cultura che attinge tanto alla narrativa popolare quanto alla letteratura moralistica e omiletica. Sembra plausibile, dunque, riconoscere all'autore una conoscenza diretta dell'Ysengrimus del monaco Nivardo, che per primo aveva fissato per iscritto una versione "renardizzata" del racconto della pesca con la coda, in cui pure figurano richiami al motivo della monacazione. $^{5}$

${ }^{4}$ Per un approfondimento sul tema si parta da Krohn 1889, Graf 1920 e Sudre 1893: 159-79.

${ }^{5}$ La familiarità che l'autore intrattiene con la (esigua) letteratura renardiana preesistente si evince anche da una fitta rete di richiami, sia allusivi che letterali, che la $b r .3$ intesse con il testo della 2. Per esempio, come annota Bonafin nella sua edizione, la battuta con cui Renart si congeda dai mercanti dopo averli derubati («Et li remanans si soit vostres!», v. 114), suona simile a quella che, nella $b r .2$, il corvo Tiecelin rivolge alla donna cui ha sottratto un formaggio («Le remanant gardes plus pres», v. 883); i mercanti, poi, rinunciando a catturare Renart, «recreant sont, arriere viennent» (v. 146), come faranno i cani che inseguono Isengrin, al termine della branche ( Li chen sont las, recreü sont», v. 504) e come fanno anche i cani che inseguono Renart nella br. 2 («recraant s'en tornent arere», v. 831). Inoltre, anche l'autore della $b r .3$ adotta un motivo che diventerà ricorrente e caratterizzante nel Roman, ovvero quello del colpo mancato, che permette a Isengrin di fuggire (ser Constant vorrebbe colpirlo ma «li colp li cola en travers», v. 481), 
È indubbio, tuttavia, che l'episodio della falsa monacazione del lupo abbia un legame assai piú lasco con gli altri due, rispetto a quello che questi ultimi intessono tra loro. Per dirla con Sudre, «la liaison entre le Moniage et la Pêche est assez indécise» (Sudre 1893: 170), al contrario di quanto accade già nel Reinhart Fuchs di Heinrich de Glîchesære, che narra i due eventi in maniera consequenziale, trascurando invece l'episodio dei carrettieri, e anche nel Van den Vos Reynaerte, in cui Renart, rievocando i tiri giocati al lupo, ricorda come lo tonsurò bruciandogli la cute («Sint dedic hem crune gheven», v. 1499) e come «sint dedic hem meerren scamp / up thijs, daer icken leerde visschen, / daer hi niene conste ontwisschen. / Hi ontfi ncker meneghen slach» [dopo gli causai altre disgrazie / sul ghiaccio, dove gli insegnai a pescare / in un posto dal quale non poteva scappare. / Lí ricevette una sonora batosta] (vv. 1504-1507, in Bowman-Besamusca 2009). ${ }^{6}$

come nella br. 2 lo aveva consentito a Renart (vv. 814-815). A questi parallelismi piú puntuali si possono aggiungere alcuni accostamenti proposti da Lucken (1989), benché in qualche caso suonino eccessivamente analogici.

${ }^{6}$ L'episodio è riportato piú estesamente nella traduzione latina del $V d v \mathrm{R}$, cioè il Reynardus vulpes (datato al 1279): dopo la tonsura, "post supra glaciem pisces huic querere suasi; / Imposita cauda sic residebat aquis; / Frigus erat magnumque gelu quod cogebat undam / Et dixi: "caveas ne moveare sedens!". / Commovet ille tamen caudam leviter, michi dicit: / "Care nepos, multis sencio piscis adest". / "Desine tunc moveas non amplius illam / Dum veniunt pisces". Hic sedet, heret aquis. / Novi lux oritur; curro, pertranseo villam, / Excito iam populos tunc moveoque canes; / Diffugio; veniunt populusque canes quoque; caudam / Nititur extrahere, non valet, heret aquis. / Cernitur, invadunt omnes; trahitur, baculatur, / Ictibus et canibus sic obiisse putant. / Unus et exemit gladium, ferit, amputa tilli / Caudam; vix, lesus, aufugit ille miser» [Poi lo persuasi a cercare i pesci sopra il ghiaccio; / immessa la coda, cosí sedeva sull'acqua; / faceva freddo e c'era un gran gelo che condensava l'acqua, / allora dissi: "bada di non muoverti restando seduto!" / Quello, però, scuote leggermente la coda e mi dice: / "Caro nipote, sento che una gran quantità di pesce si avvicina". / "Allora smetti di muoverla ancora, / finché non vengono i pesci". Lui si siede, resta bloccato sull'acqua. / Sorge l'alba del nuovo (giorno); / corro, attraverso il villaggio, / sveglio gli abitanti e aizzo i cani; / fuggo; sopraggiungono gli abitanti e anche i cani; cerca / di estrarre la coda, non riesce, rimane bloccato sull'acqua. / Viene adocchiato, lo assalgono tutti; è bersagliato, bastonato, / con le percosse e con i cani cosí pensano che sarebbe morto. / Uno estrae anche la spada, lo ferisce, gli amputa / la coda; a stento, ingiuriato, quel poverino si dilegua] (vv. 662-677, in Campbell 1859). 
La differenza è significativa dal momento che si tratta di testi piú o meno contemporanei, soprattutto nel caso del poema tedesco, composto alla fine del XII secolo - dopo il 1192, secondo Schwab (1967) - e palesemente derivato dalle branches del Roman de Renart coeve, tra cui appunto la 3, che si data agli anni 1185-1190. Un discorso simile vale per il poema fiammingo Van den Vos Reynaerte, basato sulla br. 1 del Renart e cronologicamente collocato tra il 1179 e il 1279. Qui l'avventura del furto del pesce è menzionata, ma in un luogo diverso del testo e in una variante che non corrisponde all'intreccio della br. 3: in processo, Grimbeert accusa Ysingrijn di aver imbrogliato Renart, seguendolo a distanza e divorando il pesce ch'egli man mano gettava giú da un carretto (vv. 206-216, in Bowman-Besamusca 2009).

Le motivazioni che possono aver sovrinteso a questa inserzione nella br. 3 sono sicuramente molteplici, ma forse bisogna innanzitutto sottolineare che non è implicata la ricerca di una particolare coesione strutturale del racconto, vale a dire che l'episodio in questione non sembra essere stato inserito solo per introdurre in modo logico il personaggio di Isengrin. Vari esempi della tradizione orale attestano l'esistenza di intrecci alternativi, che contemplavano la presenza del lupo fin dall'inizio (per lo piú come emulo della volpe, nel tentativo di farsi caricare anch'egli sul carretto), o che semplicemente prevedevano che subentrasse dopo il furto del pesce e venisse poi depistato dalla volpe con una falsa indicazione (come ricorda anche la br. 14). Effettivamente, l'inserzione appare piuttosto debole se la si giudica nei termini di un'aggiunta innovativa all'interno di un macro-intreccio già rigidamente testualizzato (furto ai carrettieri + pesca con la coda), ma la coerenza narrativa migliora se si prescinde da questa presupposizione di un'unità originaria degli altri due episodi e si dispongono tutti e tre i racconti su un piano idealmente paritario, senza privilegiare una combinazione precisa solo perché è tendenzialmente ricorrente.

È il confronto con la struttura del poema latino e di quello alto-tedesco a stimolare una lettura alternativa circa il riuso di questi tre segmenti narrativi nell'universo renardiano: l'assenza dell'episodio dei carrettieri dall'Ysengrimus e dal Reinhart Fuchs potrebbe suggerire che la struttura inizialmente recepita dalla tradizione renardiana prevedesse l'accostamento non del primo e del terzo episodio, bensí proprio della monacazione del lupo seguita dalla pesca con la coda. 
Questa soluzione, oltre a rendere conto dell'esclusione del primo episodio nei due testi su citati, ${ }^{7}$ spiegherebbe anche perché nella $b r .3$ è invece presente ma nella variante in cui il lupo non agisce come emulo della volpe, essendo già concepito come protagonista della finta monacazione. Forse, internamente alla branche, tale scelta adombra un primato ideale del racconto della monacazione di Isengrin sul racconto del furto delle aringhe, il quale fornirebbe solo una sorta di antefatto, automaticamente desumibile dal repertorio folklorico: in sostanza, al racconto della monacazione di Isengrin e della sua pesca nel lago ghiacciato si sarebbe, piú o meno spontaneamente, aggiunto un preambolo, costituito proprio recuperando il racconto che originariamente - e continuativamente, in alcune tradizioni popolari - era legato a quello della pesca, ovvero la storia di come la volpe si procuri il cibo sottraendolo a dei carrettieri di passaggio.

\section{TRE RACCONTI SEPARATI}

Naturalmente non è detto che la tradizione renardiana abbia seguito una direzione unitaria e univoca, ma l'alternanza con cui compare il racconto del furto, unitamente allo stretto rapporto che si ripropone tra monacazione e pesca, basta a testimoniare la forte indipendenza degli episodi che formano la $b r .3$, i quali, al tempo della sua stesura scritta, non dovevano già piú circolare necessariamente in forma aggregata, o almeno non nel milieu culturale dell'autore.

Una spia testuale dell'alto grado di separazione tra gli episodi narrati viene poi dall'ambientazione temporale del racconto. È un'incongruenza

${ }^{7}$ Un riferimento puntuale alla monacazione del lupo manca nell'Y sengrimus (circa 1148-1149), ma è un tema che attraversa tutta l'opera, dato che Isengrin è già presentato come monaco, e che comunque emerge piú volte in allusioni comiche legate ai molti patimenti che il lupo subisce. Quanto al poema tedesco, invece, l'esclusione dell'episodio potrebbe essere coerente con il taglio originariamente perseguito dall'autore, che si direbbe incentrato piú sul lupo - o comunque sul suo rapporto con Renart - che sulla volpe, da cui la definizione di Heinrich de Glîchesære come «der hat die bvch zesamene geleit / Von Isengrines arbeit» [colui che ha messo insieme le storie / della sofferenza di Isengrin], vv. 1789-1790; o della «rovina», secondo un altro testimone (vd. l'introduzione di Carla Del Zotto all'edizione italiana del Reinhart Fuchs, in Del Zotto 2007: 7-10). 
già segnalata da vari studiosi, che vede la branche - e dunque il racconto del furto del pesce - aprirsi in autunno, «en cel termine / Que li douz temps d'esté decline / Et yver revient en saison» (vv. 1-3), e chiudersi con la pesca nel lago ghiacciato in pieno inverno ( $«$ Ce fu un pou devant Noel / Que l'en mettoit bacons en sel», vv. 377-378). ${ }^{8}$

Su questo slittamento, meglio di altri si è espresso Scheidegger, considerandolo una "marca della riscrittura" che il testo ha conservato durante il processo di aggregazione dei racconti che lo costituiscono:

le vol des poissons a lieu à l'automne, Ysengrin en demande sa part et se fait tonsurer, puis il sort avec Renart, et avec la pêche au seau nous sommes juste avant Noël. Ici encore la récriture a uni la matière en enchaînant logiquement les aventures, mais il subsiste une trace, le changement de saison, de cette unification (Scheidegger 1989: 46).

D'altra parte, anche i testimoni del Roman de Renart portano il segno di una consapevole distinzione tra i tre racconti, come mostrano le rubriche che alcuni codici recano a introduzione del testo. Infatti, se i manoscritti $\mathrm{BD}$ identificano l'intera $b r .3$ con la prima avventura che la compone:

- B, c. 111e: «Cest la branche de R. com il fu getez en la charrete au pessoniers»;

- D, c. 33a: «Si comme R. fist le mort emi la voie pour decevoir charretiers qui portoient harens fres et anguiles, dont il enporta grant quantité»,

i manoscritti CM delimitano ciascuno dei tre episodi con un titolo puntuale, mostrando di intenderli come tre avventure isolate e riconoscibili:

- C, c. 5e: «Si conme R. mania le poisson aus charretiers»;

c. 6e: «Si conme renart fist ysangrin moine»;

c. $7 \mathrm{~d}$ : «Si conme R. fist peschier a ysangrin les anguiles»;

- M, c. 5a, dopo la br. 2: «Ici fenist com R. compissa les loviaus et fist y. cous et commance si com R. menga le poisson au charretiers»;

${ }^{8}$ A questo leggero sfasamento bisogna aggiungere che lo stesso esordio autunnale rappresenta un'alternativa isolata rispetto alle piú consuete coordinate temporali, primaverili o estive, delle altre branches. 
c. 6b, dopo il v. 164 della br. 3: «Ici faut si comme R. conchia les charretiers et commence de y. que R. fist moine»;

c. $7 \mathrm{~d}$, dopo il v. 376 della $b r$. 3: «Ci commance si comme R. fist peeschier a ysengrin les anguilles ou vivier» (notizie in Martin 1882-1887: VIXVI),

tanto piú che il testo di $\gamma$, cui CM appartengono, conclude l'episodio della monacazione aggiungendo due versi, assenti in entrambe le altre famiglie, in cui si dichiara esplicitamente che «ici prent ceste branche fin, / Mes encor i a d'Ysengrin» (vv. 1207-1208 dell'edizione Fukumoto-HaranoSuzuki 1983-1985).

\section{VALORI DELLA MONACAZIONE}

Nella composizione strutturale della branche - come anche nell'Y sengrimus e nel Reinhart Fuchs - emerge, dunque, un recupero piuttosto strumentale della tradizione, finalizzato a puntare il focus non sulle avventure note della volpe, ma sulla nuova componente religiosa del racconto, espressa nella comica monacazione di Isengrin. Tale è la matrice culturale da cui viene fuori questa specifica narrazione.

Dagli exempla latini di marca ecclesiastica ai proverbi, il motivo della monacazione del lupo aveva già trovato ampia diffusione, ma soprattutto «les deux siècles suivant la création de l'Ysengrimus (achevé vers 1148) ont vu s'épanouir le récit édifiant. C'est donc essentiellement dans ce type de production qu'apparaît alors le loup clerc» (Librová 2001: 204).

In tale contesto, la br. 3 non nasce tanto come una rivisitazione in chiave renardiana di una storia nota dalla tradizione orale, ma sfrutta il repertorio popolare comune come un sostrato su cui innestare il tema piú innovativo che vuole presentare. La scelta è indubbiamente incoraggiata anche dalla relativa novità del genere, che ancora trova comodo attingere dal repertorio folklorico non singoli motivi, ma intere sequenze narrative, per formulare l'intreccio di una branche. Anche in tal senso sembra corretto e utile catalogare la br. 3 all'interno di una continuation Isengrin, ${ }^{9}$ dato che il

\footnotetext{
${ }^{9}$ Come propone Zufferey (2011: 176), raggruppando le otto branches piú antiche,
} 
lupo è il vero protagonista e la sua monacazione, seguita dalla conseguente perdita della sua coda, resta l'argomento principale della vicenda.

D'altra parte, essendo funzionale a un trattamento comico del personaggio, l'evento stesso della tonsura viene in parte snaturato: conserva le apparenze di un rituale di passaggio, ma perde ogni valenza iniziatica, divenendo solo il pretesto per un inganno ai danni di Isengrin.

Innanzitutto, l'operazione subita da Isengrin, cioè la tonsura del capo, è anche quella che potrebbe riassumere la totalità dei significati simbolici della sua iniziazione: come ricorda Van Gennep, «tagliare i capelli equivale a separarsi dal mondo precedente», motivo per cui «il trattamento che subiscono i capelli rientra molto spesso nella classe dei riti di passaggio»; piú precisamente, «questo stesso rito esiste contemporaneamente nel rituale di passaggio e nel rituale di consacrazione» (Van Gennep 2019: 1467 e 147, n. 6). Tuttavia, il metodo grossolano - e beffardo - con cui tale operazione viene realizzata ne invalida sia l'intrinseca sacralità che l'effettiva validità.

Anche l'uso dell'acqua bollente richiama un contesto iniziatico, ma non propriamente cristiano: anzi, se l'acqua in sé poteva assommare alla tonsura un riferimento al battesimo, le conseguenze risibili dell'ustione che riceve il lupo farebbero pensare piú a una sorta di ordalia finita male, che non a una lustrazione.

Inoltre, va osservato che l'intera cerimonia si compie su una soglia. Renart, dall'interno dell'abitazione, svolge l'ufficio della tonsura attraverso un'apertura vicina alla porta d'ingresso; Isengrin resta fuori, limitandosi a inserire la testa nel foro predisposto:

Ö̈r poez ici biau jeu.

Renart mist l'eve sus le feu

Et la fist trestoute boillant.

Puis li est revenus devant

Et sa teste encoste de l'uis

datate 1185-1190 e precedenti alla composizione del Reinhart Fucks, in quattro tronconi: quello primitivo di Pierre de Saint-Cloud (br. 2-5a), una continuation Tibert (br. 15), una continuation Isengrin (brs. 5-3-4) e una serie di riscritture del processo riguardante l'aggressione di Renart alla lupa Hersent (brs. 1-10-6). 
Li fist metre par un pertuis:

Et Ysengrin estent le col

Renart qui bien le tint pour fol

L'eve boillant li a getée

Et sus le hasterl versée (vv. 333-342)

[Qui potete ascoltare un bello scherzo. / Renart mise l'acqua sul fuoco / e la fece diventare bollente. / Poi è tornato indietro / e gli ha fatto mettere la testa / in un foro accanto alla porta: / e Isengrino tende il collo. / Renart, che lo prendeva per stupido, / gli ha gettato l'acqua bollente / rovesciandogliela sulla nuca].

Non è un caso poiché, da una parte, in questo modo si preserva la mitica inaccessibilità di Malpertuis, sulla quale concorda la totalità delle branches renardiane. ${ }^{10}$ Dall'altra è effettivamente un luogo idoneo a tale cerimonia, in quanto «i riti compiuti sulla soglia stessa sono riti di margine» (Van Gennep 2019: 18). Neanche dopo la presunta consacrazione, però, Isengrin potrà varcare questa soglia, e il motivo è proprio che, di fatto, il rito non c'è stato: Renart lo ha solo simulato, per prendersi gioco dell'avversario.

Al contempo, benché autenticamente desideroso di intraprendere la monacazione, il lupo mostra una forte ritrosia a lasciarsi radere, palesata sia prima di ricevere la tonsura («Ysengrin commença a grondre / Quant il oï parler de rere», vv. 326-327), sia un attimo dopo (gridando a Renart che «trop grant coronne m'avez faite», v. 349), sia quando Renart lo raggiunge fuori dalla sua dimora, trovandolo ancora intento a lamentarsi:

Et vint a Ysengrin tout droit

Qui durement se complaignoit

De ce qu'il estoit si pres rez.

Ne cuir ne poil n'i est remez (vv. 369-372)

[E arrivò dritto da Isengrino / che aspramente si lamentava / d'esser stato raso cosí corto. / Né pelle né pelo gli è rimasto].

${ }^{10}$ Scorrendo le branches del Roman si noterà che nessun altro animale, ad eccezione del cugino Grimbert, riesce mai a penetrare per alcun motivo all'interno di Malpertuis. Su questo tratto tutti gli autori renardiani mantengono una solida coerenza. 
In effetti, se la rasatura è l'operazione principale e immancabile in ogni rito iniziatico, la perdita del pelo - o della capigliatura - ha anche una connotazione negativa, attestata fin dal racconto biblico di Sansone e legata alla credenza per cui la forza dell'individuo risiederebbe proprio nei suoi capelli. A livello residuale, è la stessa concezione su cui, ad esempio, si fondava la decalvatio, pena prevista nel diritto germanico, consistente in un vero e proprio scalpo, che oltre al danno fisico imponeva al condannato un marchio d'infamia permanente. ${ }^{11}$ Questa stessa funzione degradante si può ravvisare nella rasatura inflitta a Isengrin, condotta con una brutalità che è incompatibile con la cura richiesta per un taglio rituale, e che appunto sembra piú attinente a una pratica punitiva.

In questo senso, ha ragione Lucken ad accostare la tonsura di Isengrin a una vera e propria mutilazione: «il s'agit de lui enlever quelque chose, de "rooigner", de "rere", de "tondre"» (Lucken 1989: 86). In fondo, la br. 3 va proprio in questa direzione, concludendosi davvero con la perdita di un arto, quello che il lupo ha piú caro:

Se d'ilec se veult departir,

La queue li convient guerpir (vv. 449-450)

[...]

Mes la coe remest en gages:

Et molt li poise et molt li greve,

A poi son cuer de dol ne creve (vv. 496-498)

[Se di lí vuole partire, / gli conviene abbandonare la coda. / . . / Ma la coda rimase in pegno / e molto gli pesa e gli grava, / che per poco il cuore non gli scoppia dal dolore].

Non è un caso, poiché anche questa mutilazione gioca un ruolo nel definire l'umiliazione subita dal lupo, se la perdita della coda può intendersi come sostituto eufemistico di una castrazione.

${ }^{11} \mathrm{Nel}$ diritto longobardo, la decalvatio era comminata ai ladri recidivi e alle donne violente, poi a guerrieri e sovrani che si voleva disonorare. I Visigoti la applicavano anche ai presunti maghi, agli Ebrei colpevoli di blasfemia nei confronti della religione cristiana e ad altri reati della sfera matrimoniale e sessuale. Sull'argomento vd. Gasparri 1983: 141-51 e, piú specificamente sull'uso longobardo, Azzara 2013. 
Con questo valore si ritrova, ad esempio, nell'Yvain di Chretien; ${ }^{12}$ relativamente al contesto della $b r .3$, invece, l'analogia si rende piú evidente tramite un confronto con il Reinhart Fucks. Anche qui Isengrin, mutilato da ser Birtin, «ovch kleite sere her Isengrin / Den vil liben zagel sin» [si lamentò molto per la sua amatissima coda] (vv. 819-820, in Del Zotto 2007). In seguito, il lupo viene risparmiato dalle percosse mortali di alcuni monaci proprio in virtú di una confusione tra la coda mutila e una circoncisione, cosicché, spiega l'autore, «hette Ysingrin den zagel niht verlorn / noch die blatten geschorn, / in hette erhenget daz gotes her» [se Ysingrin non avesse perduto la coda / e ricevuto la tonsura, / il popolo di Dio lo avrebbe impiccato] (vv. 1021-1023). Inoltre, benché salvo, la sua condizione affligge oltremodo la consorte Hersent, che si mostra inconsolabile:

O we, ich en mag ez niht ane sin!

Mir ist leit, daz der man min

Ane zagel mvz wesen.

Wi sol ich arme des genesen? (vv. 1057-1060).

${ }^{12}$ Il cui protagonista eponimo diventa chevalier au lion proprio in seguito a un atto di mutilazione. Per salvare un leone dalle fauci di un serpente, Yvain si vede costretto a recidergli parte della sua coda, compiendo un gesto cui è stata riconosciuta una forte valenza iniziatica, legata, ancora una volta, a un simbolismo «abbastanza evidente di castrazione o ad ogni modo di domesticazione» dell'animale (Le Goff-Vidal-Naquet 1983: 130). Sull'uso metonimico della coda per indicare il pene, esempi inequivocabili sono forniti da Boccaccio nel Decameron. Nella novella di Masetto, laddove la badessa «forse estimava che egli cosí senza coda come senza favella fosse» (III, 1: 20, in Branca 1956); nelle parole dell'autore stesso, contro i suoi detrattori, che pensano «che, perché il porro abbia il capo bianco, che la coda sia verde» (IV, Intr.: 33); nell'avvertimento pronunciato dalla moglie di Giovanni Lotteringhi al suo amante: «Fantasima, fantasima, che di notte vai, a coda ritta ci venisti, a coda ritta te n'andrai» (VII, 1: 27); nel commento del narratore Dioneo alla novella appena udita: «Il buon uomo che aspettava la seguente notte di fare abbassare la coda ritta della fantasima, avrebbe dati men di due denari di tutte le lode che voi date a messer Torello» (X, 10: 2). Una conferma della sovrapponibilità di queste due immagini viene anche dalla tradizione folklorica. Nelle versioni friulane del motivo ATU 1875 [The Boy on the Bear's (Wolf's) Tail, un bambino, prigioniero in una botte, si salva perché una volpe vuole urinarvi dentro ma egli le afferra la "coda" e la strattona, cosí che la volpe inizia a correre trascinando con sé il barile, che si rompe liberando il bambino. 
[Ahimè, non posso farne a meno! / Sono addolorata che mio marito / sia senza coda. / Come farò, misera, a sopportarlo?].

Un lamento cosí accorato è poco comprensibile, a meno di non riconoscere dietro il taglio della coda un'allusione a un altro tipo di menomazione, cioè quella «della virilità di Isengrin». ${ }^{13}$ Nella $b r .3$, poi, tale è l'importanza riconosciuta a questo evento da assurgere, nelle intenzioni dell'autore, addirittura a casus belli della futura e perenne ostilità tra la volpe e il lupo, il quale

\author{
Fuiant s'en va, si se regarde, \\ Droit vers le bois grant aleüre. \\ Iloc rala et dit et jure \\ Que de Renart se vengerà \\ Ne james jor ne l'amerà (vv. 506-510)
}

[Fuggendo se ne va, guardandosi indietro, / dritto verso il bosco di gran carriera. / Colà arrivò, dicendo e giurando / che si vendicherà di Renart / e lo odierà per sempre].

È evidente, insomma, che la privazione della coda può esprimere, all'occorrenza, le stesse implicazioni disonorevoli evocate da un certo tipo di tonsura. Si comprende, allora, la scelta dell'autore di legare assieme i due episodi.

A questo punto, stante tale ambivalenza di significati relativi alla tonsura (e alla mutilazione) nella $b r .3$, occorrerà, forse, aggiungere un'interpretazione piú comica del gesto e perciò recuperare non solo i valori religiosi dell'operazione, ma anche quelli dipendenti dalla comune consuetudine, la quale associava sovente la rasatura alla pazzia.

${ }^{13}$ Del Zotto 2007: 192, sulla scorta di Schwab 1967: 65, secondo cui «diese Episode erzählt aber nichts anderes als die Entmannung des Wolfes durch die heimtückische List des Minners Reinhart, der dann auch dafür sorgt, dass die Schande durch den weiteren schaden beim Fischfang offenbar wird» [questo episodio racconta null'altro che l'evirazione del lupo tramite l'insidiosa astuzia dell'amante Reinhart, il quale si preoccupa anche che l'umiliazione diventi pubblica, attraverso l'ulteriore danno nell'episodio della pesca]. Infatti, il danno viene poi sottolineato anche dall'orso Brun, patrocinatore di Ysangrin a processo, che lo presenta come una grande offesa, tanto che il lupo «des schamt sich vaste sin lip» [patisce grande vergogna a causa del suo corpo] (v. 1381). 
Nel corpus di testi analizzato da Philippe Ménard (1977: 436), uno dei marcatori esteriori dell'individuo che ha perduto il senno è proprio portare «les cheveux tondus», e non tanto come rimedio curativo, ma come «un signe de reconnaissance et d'infamie. [...] Dans beaucoup de civilisations la tonsure est un rite d'humiliation: on renonce à certains pouvoirs, à certains privilèges; on perd une partie de sa force et de sa personnalité profonde. La perte des cheveux est vraiment une diminutio capitis» (ibi: 439).

In generale, testimonianze letterarie e giuridiche, medievali e moderne, dall'intera area europea confermano l'uso di radere il capo ai criminali e ai pazzi, probabilmente per renderli riconoscibili. ${ }^{14} \mathrm{Nel}$ solo ambito francese, la formula «tonduz comme fous» o comunque l'associazione che esprime si riscontrano, ad esempio, nella Vie Saint Thomas le Martyr («Tunduz est cumme fous», v. 1248, in Rossi 2008); ne Li proverbe au vilain («Fous dit tost tel folie / Dont on si le colie / Qu'après l'en estuet tondre», 198, vv. 1-3, in Tobler 1895); nell'Ipomedon («Lunge barbe out e res le col / Si resteit tundu cume fol», vv. 9253-9254, in Kölbing-Koschwitz 1889), nel Gaydon («Mal fu vestus, si fu estrumelez, / Et comme fox fu par lieus bertaudez», vv. 2005-2006, in Subrenat 2007); nel Merangis de Portlesguez. («trop li mesavient / Sa teste qu'il avoit tondue. / Il ne li faut fors la maçue / A sambler fol», vv 4943-4946, in Szkilnik 2004); nel récit 10 (Fou) della Vie des Pères («q'un fol tondu, simple, sanz guile / Troverez, ki a non Felis», vv. 4854-4855, in Lecoy 1987, t. 1); nel Roman des deduis («Je di c'om devroit celui tondre / Comme fol», vv. 5488-5489; «Mieux eust esté d'un fol tondu / Proposé que ne l'ay sceu faire», vv. 10522-10523, e «Maiz il n'est fol en croiz tondu», v. 9196, in Blomqvist 1951); ne La Folie Tristan di Berna («Haut fu tonduz, lonc ot lo col, / A mervoille sambla bien fol», vv. 152-153, e «Por vos s'est tonduz conme fol», v. 363, in Lecoy 1994). ${ }^{15}$ A proposito di quest'ultimo testo, anche Ménard, confron-

${ }^{14} \mathrm{Vd}$. Tilander 1958. Un esempio fiammingo del XIV sec. specifica trattarsi di «een brede crune toten oren» [una grande tonsura fino alle orecchie] (in Tilander 1958: 225), come quella che Renart pratica al lupo Primaut nella $b r$. 14, quando «la corone dusqu'as orelles / Li a fete» (vv. 398-399). Sulle tipologie di tonsura praticate nel Medioevo e sui loro significati, vd. Gross-Thibault-Schaefer 1995, che offre anche un ricco dossier iconografico.

${ }^{15}$ L'elenco, non esaustivo, proviene da Tilander 1958: 227-8, di cui ho aggiornato 
tando le occorrenze del Tobler-Lommatzsch (10: 376-7, s. v. tondre), osserva che una formula come "haut tondu" "s'emploie aussi bien pour les fous que pour les clercs tonsurés» (Ménard 1977: 437). ${ }^{16}$

La scelta di compiere su Isengrin una finta tonsura, dunque, è finalizzata a una sua denigrazione, poiché egli viene tacitamente deriso da Renart con l'applicazione di un contrassegno che parrebbe seriamente religioso ma che può alludere a una sua condizione di insania, tanto che lo stesso Renart «bien lo tint pour fol» (v. 340). Del resto, non sarebbe una novità. I gabs con cui Renart saluta gli avversari insistono sempre sulla risibilità della loro umiliazione: basti pensare ai motteggi rivolti all'orso Brun nella $b r .1$, quando, perduta la pelle del capo per essere rimasto incastrato in un albero, si sente chiedere da Renart «de quel ordre voles vos estre / Que roge caperon portes?» (vv. 698-699). Piú avanti, il rossore diventa lo stesso marchio di riconoscimento dell'orso, definito in un elenco come «Bruns qui la teste ot vermeille» (v. 1208). In un'altra branche Renart, dopo aver causato a Isengrin uno scalpo, per sbeffeggiarlo in vista di un duello contro di lui, si fa rasare la testa e radere il pelo: «Haut fu rooingnies et tondus, / Et col et barbe se fist rere / Por le despit de son conpere» (br. 6, vv. 896-898). Come nel caso di Brun, anche per il lupo si conia un appellativo ad hoc, cioè «Ysengrin li chenu» (in un'aggiunta dei mss. CHM, v. 17).

Peraltro, l'autore della $b r .3$ specifica che dopo l'operazione «ne cuir ne poil n'i est remez» (v. 372), da cui sembra di intendere che Isengrin

alcuni riferimenti, segnalando un'edizione piú moderna nel caso della Vie Saint Thomas, della Folie Tristan, del Gaydon, del Merangis, della Vie des Pères. Da quest'ultimo testo si può citare anche un'altra occorrenza precedente, legata allo stesso personaggio di Felix il quale, suggestionato dalla vista di un cimitero, decide che «en l'ordre de Citeaz morrai, / Et folz tonduz et folz pelez / Vuel estre en cest monde apelez» (10, Fou, vv. 44434444). Quanto alla prima citazione della Folie di Berna, ancor piú calzante è la variante attestata nel frammento di Cambridge, che sostituisce «lonc» con «reis», cioè appunto 'rasato': «Aut fu tundu, reis vot le cole», v. 3, in Lecoy 1994: 35.

${ }^{16} \mathrm{Vd}$. anche la voce bertouder del TL, che offre in sequenza una citazione legata al valore di follia intrinseco nel taglio dei capelli e una legata alla monacazione: la prima è quella del Gaydon già citata, la seconda è tratta da uno dei Miracles de Notre Dame di Gautier de Coincy, quello D'un chevalier qui amoit une dame e recita: «Moines tondus et bertaudés / Seroie anchois» (TL 1: 935). 
abbia ricevuto, se non uno scalpo, quantomeno una tonsura totale del capo. Anche questa è una soluzione che diverge dalla prassi clericale, ${ }^{17}$ applicandosi piuttosto ai pazzi, per i quali fungeva appunto da stultitiae indicium (vd. Du Cange 1883: 128-9, s. v. capilli).

\section{LA TONSURA NELL'YSENGRIMUS}

La preminenza delle implicazioni comiche della tonsura non è un'esclusiva della $b r .3$, poiché anche nell'Y sengrimus l'ironia ai danni del lupo si esprime in una serie di allusioni alla pratica della monacazione: «le traitement subi par le loup lors de la pêche est sans cesse présenté ironiquement comme un renouvellement de sa tonsure [...] et la queue coupée une nouvelle nomination» (Lucken 1989: 77). I passi cui fa riferimento Lucken sono quattro. Nel primo, Renart informa beffardamente Isengrin dell'arrivo di un prete intenzionato a malmenarlo:

Et tibi neglectam pensat renovare coronam,

Discessusque tui vult abolere nefas.

Quanta sit impietas hinc me fugisse, probabis,

Cum fuerit capitis silva putata tui;

Tunc vere quia plena dei sit copia, dices,

Cum benedicta tuum sparserit unda caput

(Liber I, vv. 857-862, in Voigt 1884)

[E ha intenzione di rinnovarti la tonsura trascurata, / e vuole cancellare il crimine della tua diserzione. / Quanta empietà ci sia nel fatto che io sia fuggito da qui, lo stimerai tu, / quando sarà stata sfrondata la foresta del tuo capo; / allora sí che dirai come i beni di Dio siano abbondanti, / quando il tuo capo sarà bagnato con l'acqua benedetta].

Nel secondo, è Aldrada, personaggio femminile del gruppo degli assalitori, a preannunciare sardonicamente al lupo di voler decapitarlo:

${ }^{17}$ Il taglio integrale dei capelli era previsto nel caso della cosiddetta "tonsura di San Paolo", diffusa però molti secoli prima e principalmente nel monachesimo orientale. 
Non ego curarem, si quid prodesset habenti,

Tollere, sed senuit, sed probitate caret,

Et capite exempto levior fit sarcina trunci,

Non est, cur teneas amplius, ergo metam;

Ne crebro indigeat tonsore corona recrescens,

Rado tibi pariter colla caputque semel (Liber II, vv. 29-34)

[Non mi preoccuperei di rimuoverla, se giovasse a qualcosa al proprietario, / ma è invecchiata, difetta di onestà, / e poi, tolta la testa, diventa piú leggero il peso del corpo. / Non c'è motivo che tu la tenga piú a lungo, perciò la taglierò; / e affinché la tonsura, ricrescendo, non richieda di continuo un barbiere, / ti rado ugualmente il collo e la testa insieme].

Nel terzo caso, Renart deride la ferita alla testa che Aldrada ha procurato a Isengrin, fingendo di scambiarla per un capo di vestiario:

Membra quis hoc scisso texit tibi regia sacco?

Non habuit nostros talis amictus avos!

Amodo piscandi studium sectabere forsan,

Et piscatores frigora saepe gravant,

Ergo gravesne ista ventos crebrosque cuculla

Assultus gelidae pellere credis aquae? (Liber II, vv. 193-198)

[Chi ti coprí le regali membra con questo sacco lacero? / Tale abito non fu proprio dei nostri avi! / Forse inseguirai ora la tua passione per la pesca, / e spesso i freddi affaticano i pescatori, / per cui credi di respingere con questo cappuccio $^{18}$ / i venti intensi e l'assalto ripetuto dell'acqua gelida?].

Infine, poco piú avanti, Renart ricorre alla stessa ironia per giustificare il suo allontanamento, quando Isengrin aveva dovuto subire la mutilazione della coda:

Denique credidimus caudam truncantibus illis,

Quod fieres abbas claustra novena super,

Ut tot praebendis, ne paupertate gravante

Rursus suffugeres, efficerere satur.

Quod si, cauda tibi cur sit mutilata, requiris,

${ }^{18}$ Il termine cuculla indica anche il cappuccio dell'abito monacale e, per estensione, la condizione religiosa. 
Istius officii congrua causa fuit:

Luxus opes sequitur, sibi quisque fit utilis abbas, Sanctior est, quisquis pinguior esse potest,

[...]

Qui sapit, est sapiens: tu, pauper, multa vorasti,

Ingluviem, dives, prosequerere magis,

Et crassus fieres abbatum more proborum,

Idcirco utiliter cauda resecta tibi est (Liber II, vv. 239-252)

[Quindi credemmo, troncandoti quelli la coda, / che saresti stato nominato abate di nove conventi, / affinché, saziato con tante offerte, avresti fatto in modo / di non fuggire ancora sotto il peso della povertà. / Pertanto, se domandi come mai ti fu mozzata la coda, / la causa opportuna fu di questo incarico: / con il potere viene la dissolutezza, ciascun abate mira al proprio utile, / chi piú può essere grasso, piú è santo, / . . / Chi ha gusto, è sapiente: ${ }^{19}$ tu, da povero, mangiasti con voracità tante cose, / da ricco, avresti perseguito ancora di piú la ghiottoneria / e saresti diventato grasso come un onesto abate, / perciò utilmente la coda ti fu recisa].

La circostanza non stupisce, dato che anche la letteratura moralistica aveva buona dimestichezza con la figura del lupo monaco. In questo ambito, un'attestazione interessante è quella del predicatore Odone di Cheriton, che in un sermone In Circumcisione Domini sfrutta proprio l'esempio del lupo per inserire una parabola sui falsi religiosi:

Dicitur: lupus, ut possit carnibus agninis saturari, fecit se tonderi et monachus factus est; deficientibus carnibus, cucullam reliquit et ad silvam reversus est. Verumtamen, qui artam subeunt religionem, praesumatur quod, ut [pro] Domino in angustiis, non in deliciis, militent, se circumcidunt (in Hervieux 1896: 270, citato da Librová 2001: 206-7)

[Si dice che il lupo, per potersi rimpinzare con carni di agnelli, si fece radere e fu ordinato monaco; poiché le carni scarseggiavano, lasciò il cappuccio e tornò nel bosco. Tuttavia, quelli che sopportano una vita religiosa angusta, presumendo di militare per il Signore nelle difficoltà, non nei piaceri, si circoncidono].

${ }^{19}$ La traduzione offusca il gioco di parole su sapĕre, che vale sia "avere il senso del gusto", sia "essere saggio". 
La brevità del richiamo tradisce la sua notorietà (marcata anche dall'impersonale dicitur), per la quale, in tutti i testimoni noti, «les copistes ont jugé suffisant de rappeler succinctement sa trame», lasciando «aux prédicateurs de l'amplifier à leur gré» (Librová 2001: 207). Cosí fa lo stesso Odone in una sua favola, De lupo qui voluit esse monachus.

In effetti, questo del lupo monaco è un motivo abbastanza diffuso, che ricorre spesso in combinazione con un altro, quello del lupo a scuola, ${ }^{20}$ di cui esempio particolarmente noto è la favola De presbytero et lupo di Maria di Francia:

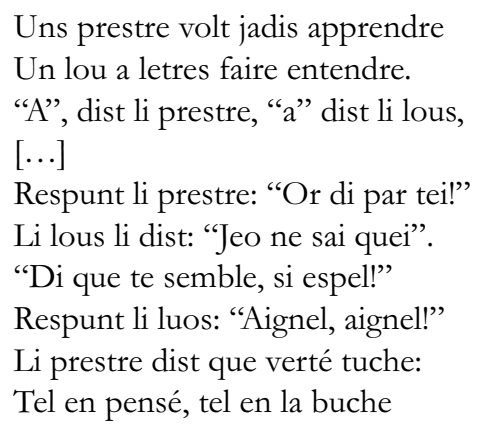

(in Morosini 2017: 147-8, traduzione a fronte)

[C'era una volta un prete che voleva insegnare / e far capire l'alfabeto ad un lupo: / "A", disse il prete, "a", disse il lupo, / . . / Il prete rispose: "Ora dillo da solo!" / Il lupo gli disse: "Non so che cosa". / "Dici ciò che ti pare, dai!" / Il lupo rispose: "Agnello!" / Il prete gli disse che era proprio vero il detto: / tale pensiero, tale parola].

La stessa riluttanza ad apprendere correttamente le formule liturgiche si riscontra nel lupo della favola di Odone, che infatti comprende già entrambi i motivi:

Ysemgrinus [sic $]^{21}$ semel voluit esse monachus. Magnis precibus optinuit quod Capitulum consensit; coronam, cucullam et cetera monachalia suscepit. Tan-

${ }^{20}$ Una sintetica panoramica sull'argomento si può trovare in Del Zotto 2001: 28892.

${ }^{21}$ Hervieux, basandosi sul ms. Cambridge, Corpus Christi College, Parker Library, 441, mette a testo una variante anomala del nome Ysengrimus, ma buona parte della 
dem posuerunt eum ad litteras; debuit addiscere Pater noster, et semper respondit Agnus vel Aries. Docuerunt eum ut respiceret ad Crocifixum, ad sacrificium, et ille semper direxit oculos ad arietes. Sic plerique fiunt monachi... (in Hervieux 1896: 195)

Una volta Isemgrino volle essere monaco. Con molte suppliche ottenne che il Capitolo acconsentí: assunse la tonsura, il cappuccio e gli altri ornamenti monacali. Infine, lo disposero alle lettere; dovette imparare bene il Padre Nostro, e sempre rispondeva Agnello o Ariete. ${ }^{22}$ Gli insegnarono a rivolgere l'attenzione al Crocifisso, all'offertorio, ma lui dirigeva sempre gli occhi verso gli arieti. Cosí fa la maggior parte dei monaci...].

In piú, non mancano tracce di una conoscenza diretta dell'Y sengrimus da parte di Odone, dal momento che il lupo protagonista della favola porta lo stesso nome e la sua fallimentare educazione religiosa è descritta in termini simili a quelli usati da Nivardo:

Ysengrimus erat frater, dudumque sepulti

Sumere presbiteri poscitur ipse locum;

Ille rogat, quod opus soleat patrare sacerdos?

Pascere berbices anne parare dapes?

At typice fratres ovibus dixere tuendis

Praefore presbiterum, paruit ille libens.

Continuo "Dominus vobiscum!" dicere iussus,

Ysengrimus ovans, "cominus", inquit, "ovis!"

[...]

Dumque docent "amén" quasi graecum, accentuat "ágne"

(Liber V, vv. 541-549)

[Isengrin era frate e gli viene chiesto di assumere / la posizione di un sacerdote sepolto di recente; / lui domanda: "quale occupazione era solito svolgere il sacerdote? / Pascolare i montoni o allestire i banchetti?” / Ma a gesti i fratelli dissero che il presbitero / sovrintendeva alla custodia delle pecore, egli obbedí compiacente. / Immediatamente comandato di dire "Dominus vobiscum!", / Isengrin, gioendo, dice "cominus, ovis!"23 / . . / E quando gli insegnano "amén”, alla greca, pronuncia "agnéllo”].

tradizione testimoniale della favola condivide, con minime oscillazioni, la lezione "Ysengrinus», ovvero quella derivata da una latinizzazione del francese Ysengrin (vd. l'edizione Voigt 1878: 117).

${ }^{22}$ Sottinteso: in luogo di "Amen".

${ }^{23}$ Letteralmente: «comandato di dire "il Signore sia con voi!", Isengrin, gioendo, 
Insomma, l'intuizione di sfruttare, episodicamente, la comicità suscitata dalle possibili connotazioni religiose del lupo è ampiamente suggerita da materiali preesistenti, tanto piú che anche Isengrin, nella $b r .3$, ostenta una conoscenza maldestra del latino, esclamando «Nomini dame» in luogo di "in nomine Domini" (v. 240). Eppure, i modi in cui questa immagine è stata recuperata nel Roman tradiscono un'inedita artificiosità. Mentre il lupo di Nivardo diviene un utile strumento per un'invettiva contra monachos, poiché già nelle premesse, «si Isengrin peut facilement devenir moine, c'est parce que les moines sont eux-mêmes des loups, rapaces et voraces», ${ }^{24}$ nel Roman de Renart la monacazione è un mero pretesto narrativo e la $b r .3$ non fa eccezione: semplicemente, «la quête d'Isengrin affamé vient relancer le récit en menaçant cette abondance alimentaire» che Renart si era procurato (Bellon 2002: 223), per cui tocca ora a quest'ultimo escogitare un nuovo engin per tutelarsi.

In sostanza, il motivo del moniage è ovviamente compatibile con la costante tendenza anticlericale che scorre in molte branches del Roman, ma non sembra direttamente concepito in sua funzione.

\section{Morfologia DELLA BRANCHE}

Queste corrispondenze (insieme ad altri richiami a catena tra vari testi, illustrati nel contributo di Librová) confermano l'esistenza di un motivo letterario ben standardizzato, consistente in un travestimento del lupo con abiti monacali, che parrebbe procedere di pari passo con la menzione dei suoi insuccessi nella catechesi cristiana, e che deve aver avuto fortuna, in questa forma, soprattutto presso scrittori moralisti e predicatori, data la facile adattabilità del modello a narrazioni moraleggianti o ad exempla, fino a divenire proverbiale. ${ }^{25}$

dice "faccia a faccia, agnello!"», alludendo alle possibilità che gli si offrono per avvicinare indisturbato il bestiame e divorarlo.

${ }^{24}$ Cosí anche Batany 1989: 124, secondo cui «Ysengrin est pour les moines un "miroir", un speculum, comme on disait alors». Per una trattazione diffusa sui casi di moniage nel Renart e sulle differenti forme di satira contro i religiosi sviluppate nella letteratura precedente e coeva, vd. l'intero capitolo (pp. 109-41).

${ }^{25}$ La stessa Maria di Francia, in un'altra favola (De lupis), ripropone il tema in una 
Pertanto, diviene chiaro che anche la monacazione di Isengrin non è affatto avulsa da una precisa tradizione narrativa - stavolta di matrice clericale, ma condivisa certamente dal vasto pubblico,$-{ }^{26}$ cui l'universo renardiano ha presto attinto in varie misure. In modo piú completo e puntuale nell'Y sengrimus; in modo piú parziale e fugace nella $b r$. 3. Per quest'ultima, si può immaginare che il recupero del motivo del lupo chierico, già centrale nel poema latino, sia stato incoraggiato anche dalla facilità con cui consentiva di sviluppare un nuovo intreccio renardiano, in un momento in cui il genere si andava ancora codificando (non a caso, quindi, ancora nel contesto di una continuation Isengrin).

Riassumendo, la br. 3 veicola due diverse tipologie di motivi etnici, di cui uno derivante da una tradizione folklorica prevalentemente orale, ovvero il racconto della pesca con la coda, al quale si aggiunge quello del furto ai carrettieri; l'altro sviluppato principalmente attraverso fonti scritte, benché in costante osmosi con la cultura popolare, propria di tutti coloro ai quali questi testi si rivolgevano (per esempio, i fedeli cui erano destinate le prediche di Odone etc.). Il punto, però, è che l'autore della branche sembra essere partito proprio da quest'ultimo motivo, già presente nell'universo letterario renardiano, per compilare un racconto relativamente nuovo. Lo ha costruito semplicemente sfruttando quel materiale folklorico già testualizzato in forma renardiana nell'Y $Y$ sengrimus (il racconto della pesca con la coda) e arricchendo l'intreccio con un preambolo desunto da quello stesso repertorio tradizionale, ovvero il racconto del furto del pesce, che sovente accompagnava quello della pesca. Si noti, infatti, che nella $b r .3$ la pesca nel lago ghiacciato perde anche la motivazione che la accompagnava

forma sentenziosa: «Ki sur le luo metreit bon mestre, / Ki doctrinast a estre prestre, / Si sereit il tuz dis gris luos, / Fel e engrés, laiz e hisdous» [Anche se il lupo avesse un buon maestro / Che gli insegnasse a fare il prete, / Resterebbe sempre e comunque un lupo grigio, / Furbo e crudele, brutto e sporco] (in Morosini 2017: 124-5).

${ }^{26}$ Come prova la gran quantità di rappresentazioni di animali in vesti monacali che affollano le chiese medievali. Occorre comunque precisare che la maggioranza delle raffigurazioni predilige il tema della volpe, piú che del lupo, in abiti religiosi, che vanno dal pellegrino, al monaco, all'eremita, al vescovo (quest'ultimo, «peculiarly English», secondo Varty 2000: 439). Il piú delle volte in atteggiamento predicatorio, riflesso, probabilmente, della notorietà del proverbio: "quando la volpe predica, guardatevi, galline", come suggerisce Varty (ibi: 435). 
da sempre, cioè rifornire di cibo il lupo, per diventare solo un'ulteriore prova che Isengrin dovrà superare al fine di ottenere l'ammissione al convento. Lo dichiara apertamente Renart, quando spiega al compare che «iceste premeraine nuit / Vous convient estre en espreuve: / Que li sains ordres le nous rueve» (vv. 356-358).

È vero che esiste un'ipotesi piú semplice di ricostruzione della genesi della $b r$. 3: l'autore avrebbe imitato la tradizione folklorica che prevedeva i due motivi $A T U 1$ e $A T U 2$ in successione lineare e avrebbe solamente approfondito la circostanza in cui il lupo chiede alla volpe parte del suo bottino, costruendoci su il racconto di una monacazione. Eppure, questa interpretazione sembra viziata da un pregiudizio che tende a sovrastimare la rigidità dell'intreccio "furto + pesca" nella tradizione europea, o quantomeno francese, di fine XI secolo, contemporaneamente sottostimando il contributo dell'autore e dei suoi riferimenti culturali nella stesura della branche. Il racconto della pesca con la coda risulta, invece, formalmente dipendente dal tema della monacazione del lupo, e ancor piú il suo complemento, cioè il racconto di come la volpe sottrae il pesce ai carrettieri, trova senso nell'economia del récit a partire dalla presenza del primo, fornendo solo marginalmente un pretesto per lo sviluppo della trama.

In sostanza, l'esistenza di un intreccio popolare articolato in tre momenti, ovvero: 1. La volpe ruba del pesce da un carretto; $>2$. il lupo, affamato, la incontra e le chiede una porzione; $>3$ la volpe, per danneggiarlo, induce il lupo a procurarsi il pesce da sé, pescando in un lago ghiacciato, fornisce, in realtà, solo uno spunto all'autore della $b r .3$, il quale organizza la propria narrazione avendo in mente innanzitutto il modello letterario dell'Y sengrimus e rivolgendosi al folklore in seconda istanza, sulla scia già tracciata da Nivardo. Pertanto, è a partire dalla monacazione di Isengrin che va ricostruita la genesi della branche, nonostante il tono complessivo del racconto tenda poi inevitabilmente a concentrarsi sulle azioni bricconesche di Renart. L'autore avrà allora concepito la sua storia in quest'ordine: 1 . Il lupo Isengrin, affamato, giunge alla dimora della volpe per chiedere del cibo, attratto dall'odore del pesce che Renart sta cucinando. La menzione del pesce è funzionale a introdurre il motivo della monacazione, poiché Renart spiega che i monaci cui sta dando ospitalità - e ai quali si è unito - non possono mangiare altro. ${ }^{27}$ Cosí, per averlo, Isengrin

\footnotetext{
${ }^{27}$ Vv. 253-260: «“Or me dites, mangiez vous char?” / Et dist Renart: “Ce est
} 
accetta di unirsi a loro e ricevere una tonsura; $>2$. la circostanza richiama il motivo della pesca nel lago ghiacciato, che qui viene inserita come una prova imposta al lupo per completare la monacazione (e il cui risultato, come si è visto, veicola lo stesso valore comico e degradante della tonsura); $>$ 3. la pesca nel lago ghiacciato rievoca, a sua volta, il racconto di come la volpe si era procurata il pesce, per cui si aggiunge in apertura l'episodio (nient'affatto essenziale ai fini della trama) di Renart che ruba il pesce da un carretto.

Questa diversa prospettiva permette di riconoscere nella $b r .3$ un ottimo esempio del modo in cui una tradizione folklorica è stata spesso recepita e riproposta in molti testi renardiani, vale a dire con una disinvoltura estrema, che può toccare non solo i contenuti, come accade in certi casi, ma anche la morfologia degli intrecci, per adattarli alle superiori esigenze narrative degli autori.

Mauro de Socio

(Università degli Studi di Macerata)

\section{RIFERIMENTI BIBLIOGRAFICI}

\section{LETTERATURA PRIMARIA}

Decameron (Branca) $=$ Giovanni Boccaccio, Decameron, a c. di Vittore Branca, Torino, UTET, 1956.

Reynardus vulpes (Campbell) = Boudewijn de Jonghe [Balduino il giovane], Reynardus vulpes, poëma ante annum 1280 a quodam Baldwino e lingua teutonica translatum, recudi curavit M.F.A.G. Campbell, Hagae Comitis, Martinum Nijhoff, 1859.

eschar". / "Que menjuent donc vostre moine?" / "Jel vous dirai sanz nule essoine. / Il menjuent fourmages mous / Et poissons qui ont les gros cous. / Saint Beneoit le nous commande / Que ja n'aions peior viande"» ["Ditemi, mangiate carne?" / "Prendete in giro?" disse Renart. / "Che mangiano allora i vostri monaci?” / "Ve lo dirò senza indugio. / Mangiano formaggi teneri / e pesci dal collo largo. / San Benedetto ce lo comanda / di non mangiare cibo peggiore"]. 
Gaydon $($ Subrenat $)=$ Gaydon, chanson de geste du XIIIe siècle. Édition, traduction (en collaboration avec Andrée Subrenat), présentation et notes par Jean Subrenat, Leuven, Peeters, 2007.

Le roman des deduis (Blomqvist) $=$ Gace de la Buigne, Le roman des deduis. Édition critique d'après tous les manuscrits, par Åke Blomqvist, Stockholm, Almqvist och Wiksell · Paris, Thiébaud, 1951.

Reinhart Fucks (Del Zotto) = Heinrich der Glîchesære, La volpe Reinhart, a c. di Carla Del Zotto, Milano, Carocci, 2007.

Hue de Rotelande (Kölbing-Koschwitz) = Hue de Rotelande, Ipomedon. Ein franæösischer Abenteuerroman des 12. Jahrbunderts. Als Anhang zu der Ausgabe der drei englischen Versionen zum erster Male, hersg. von E. Kölbing und E. Koschwitz, Breslau, Koebner, 1889.

La Folie Tristan $($ Lecoy $)=$ Les deux poèmes de «La Folie Tristan», éd. par Félix Lecoy, Paris, Champion, 1994.

Li proverbe au vilain $($ Tobler $)=$ Die Sprichwörter des gemeinen Mannes, altfranzösische Dichtung nach den bisher bekannten Handschriften, hersg. von Adolf Tobler, Leipzig, Hirzel, 1895.

Favole (Morosini) = Maria di Francia, Favole, a c. di Roberta Morosini, Roma, Carocci, [2006] 2017.

Ysengrimus $($ Voigt $)=$ Nivardo di Gand, Y sengrimus, hersg. von Ernst Voigt, Halle a S., Verlag der Buchhandlung des Weisenhauses, 1884.

Odone di Cheriton (Hervieux) = Léopold Hervieux (éd. par), Les fabulistes latins depuis le siècle d'Auguste jusqu'à la fin du moyen âge, t. IV, Eudes de Cheriton et ses dérivés, Paris, Firmin-Didot et c.ie, 1896.

Odone di Cheriton (Voigt) $=$ Ernst Voigt (hersg. von), Kleinere lateinische Denkmäler der Thiersage aus dem zwölften bis vierzebnten Jabrbundert, Strassburg, Trübner, 1878.

Meraugis de Portlesguez (Szkilnik) = Raoul de Houdenc, Merangis de Portlesguez: Roman arthurien du XIII e siècle, publiè d'après le manuscrit de la Bibliothèque du Vatican, édition bilingue, publication, traduction, présentation et notes par Michelle Szkilnik, Paris, Champion, 2004.

Roman de Renart (Bonafin) = Il Romanzo di Renart la volpe, a c. di Massimo Bonafin, Alessandria, Edizioni dell'Orso, [1999] 2004.

Roman de Renart (Fukumoto-Harano-Suzuki) = Le Roman de Renart, édité d'après les manuscrits C et M, par Naoyuki Fukumoto, Noboru Harano et Satoru Suzuki, Tokyo, France Tosho, 1983-1985, 2 voll.

Roman de Renart (Martin) = Le Roman de Renart, éd. par Ernest Martin, Strasbourg, Trübner, 1882-1887, 3 voll.

Vie des Pères (Lecoy) $=$ La Vie des peres, éd. par Félix Lecoy, Paris, Société des anciens textes français, 1987, 3 voll. 
Vie Saint Thomas le Martyr (Rossi) = La Vie Saint Thomas le Martyr, edizione critica a c. di Carla Rossi, Alessandria, Edizioni dell'Orso, 2008.

Van den vos Reynaerde (Bowman-Besamusca) = Willem (?), Of Reynaert the fox. Text and Facing Translation of the Middle Dutch Beast Epic Van den vos Reynaerde, ed. by André Bouwman and Bart Besamusca, Amsterdam, Amsterdam University Press, 2009.

\section{LETTERATURA SECONDARIA}

ATU $=$ Hans-Jörg Uther, The Types of International Folktales: a Classification and Bibliography. Based on the system of Antti Aarne and Stith Thompson, Helsinki, Suomalainen Tiedeakatemia, 2004, 3 voll.

Azzara 2013 = Claudio Azzara, Pene «infamanti» nelle leggi dei Longobardi, in Claudio Azzara et alii (a c. di), Historiae. Scritti per Gherardo Ortalli, Venezia, Edizioni Ca'Foscari, 2013: 11-22.

Batany 1989 = Jean Batany, Scène et coulisses du «Roman de Renart», Paris, SEDES, 1989.

Bellon 2002 = Roger Bellon, L'animal devenu moine : l'emploi du motif du moniage dans l'épopée animale, de l' YYsengrimus» latin au «Renart» français, in Pierre Nobel (éd. par), Formes et figures du religieux au Moyen Âge, Besançon, Presses Universitaires Franc-Comtoises, 2002: 217-31.

Del Zotto 2001 = Carla Del Zotto, Exempla della tradizione esopica e zooepica nel «Wälscher Gast» di Tommasino di Cerclaria, «Rivista di cultura classica e medioevale» 43/2 (2001): 267-92.

Gasparri 1983 = Stefano Gasparri, La cultura tradizionale dei Longobardi. Struttura tribale e resistenze pagane, Spoleto, CISAM, 1983.

Graf 1920 = Adolf Graf, Die Grundlagen des «Reineke Fuchs»: eine vergleichende Studie, Helsinki, Suomalainen Tiedeakatemia, 1920.

Gross-Thibault-Schaefer 1995 = Angelika Gross, Jacqueline Thibault-Schaefer, Sémiotique de la tonsure, de l'"insipiens" à Tristan et aux fous de Dieu, in Aa. Vv., Le clerc au Moyen Age, Aix-en-Provence, CUERMA, 1995: 244-75.

Harano 1999 = Noboru Harano, Le texte $\gamma$ de la branche III du «Roman de Renart», «Reinardus» 12 (1999): 35-44.

Krohn 1889 = Kaarle Krohn, Bär (Wolf) und Fuchs: eine nordische Tiermärchen kette. Vergleichende Studie, «Journal de la Société Finno-ougrienne» 6 (1889): 1-132.

Le Goff-Vidal-Naquet 1983 = Jacques Le Goff, Pierre Vidal-Naquet, Abbozzo di un'analisi di un romanzo cortese, in Jacques Le Goff, Il meraviglioso e il quotidiano nell'Occidente medievale, Milano · Bari, Laterza, 1983: 101-43.

Librová $2001=$ Bohdana Librová, Le moniage du loup: du récit à l'expression figée, 
«Reinardus» 14 (2001): 203-21.

Lucken $1989=$ Christopher Lucken, Aus grans Pescheurs eschapent les anguilles, «Littérature» 74 (1989): 76-90.

Ménard 1977 = Philippe Ménard, Les fous dans la société médiévale. Le témoignage de la littérature au XIIe et an XIIIe siècle, «Romania» 98 (1977): 433-59.

Rychner 1969 = Jean Rychner, La critique textuelle de la branche III (Martin) du «Roman de Renart» et l'édition des textes littéraires francais du Moyen Âge, «Bulletin d'information de l'Institut de Recherche et d'Histoire des Textes» 15 (1969): 121-36.

Scheidegger 1989 = Jean R. Scheidegger, Le «Roman de Renart» ou le texte de la dérision, Genève, Droz, 1989.

Schwab 1967 = Ute Schwab, Zur Datierung und Interpretation des «Reinhart Fuchs». Mit einem textkritischen Beitrag von Klaus Düwel, Neapel, Cymbra Verlag, 1967.

Sudre 1893 = Léopold Sudre, Les sources du «Roman de Renart», Paris, Bouillon, 1893.

Tilander 1958 = Gunnar Tilander, O uso de rapar a cabeça aos loucos e a etimologia do port., esp., it. tonto, rom. tînt, tont (louco), «Revista de Portugal. Serie a, lingua portuguesa» 33/164 (abril 1958): 223-32.

$T L=$ Adolf Tobler, Erhard Lommatzsch, Alffranzösisches Wörterbuch, Berlin, Weidmann, 1915-2002.

Van Gennep 2019 = Arnold Van Gennep, I riti di passaggio (1909), trad. di Maria Luisa Remotti, Torino, Bollati Boringhieri, 2019.

Varty $2000=$ Kenneth Varty, Masks of the fox religious in Medieval and Renaissance English art, «L'immagine riflessa» 9/1-2 (2000): 429-45.

Voigt 1879 = Ernst Voigt, Odo de Ciringtonia und seine Quellen, «Zeitschrift fiir deutsches Altertum» 23 (1879): 283-307.

Zufferey 2011 = François Zufferey, Genèse et tradition du roman de «Renart», «Revue de linguistique romane» 75 (2011): 127-89.

RiAsSUNTO: Se l'autore della branche 3 del Roman de Renart attinge ampiamente al patrimonio narrativo orale per la stesura del suo racconto, l'episodio della monacazione del lupo proviene, però, da una tradizione affatto diversa, letteraria e di matrice religiosa. Tutt'altro che accessoria, si proverà ad argomentare che proprio da tale inserzione dipende l'intera articolazione della branche, ovvero sia la scelta del materiale folklorico selezionato (cioè i due racconti del "furto del pesce dal carretto" e della "pesca con la coda"), sia la sua riproposizione in veste renardiana.

PAROLE CHIAVE: Renart, Isengrin, branche 3, tonsura, moniage. 
ABSTRACT: Besides the author of Branch 3 of the Roman de Renart draws extensively from the oral narrative patrimony for the writing of his tale, nevertheless the episode of the wolf taking monastic vows comes from a quite different tradition, which is literary and religious. Far from being incidental, we try to argue that the entire articulation of the Branch depends just on such insertion, both as regards the choice of the folkloric material (that is the two tales of the "theft of the fish from the cart" and the "fishing with the tail"), and its reinterpretation in a Renardian guise.

KEYWORDS: Renart, Isengrin, Branch 3, tonsure, moniage. 